\title{
Speciality Grand Challenges: Blockchain
}

\author{
Philipp Sandner ${ }^{1 *}$ and Philipp M. Schulden ${ }^{2}$ \\ ${ }^{1}$ Frankfurt School Blockchain Center, Business Administration at Frankfurt School of Finance \& Management, Frankfurt am \\ Main, Germany, ${ }^{2}$ Frankfurt School Blockchain Center, Frankfurt School of Finance \& Management, Frankfurt am Main, \\ Germany
}

Keywords: blockchain, governance, loT, regulation, scalability, crypto assets, DLT

The distributed ledger technology promises to change many long-standing economic and financial concepts through its unique properties of transparency, immutability, and distribution. Not only universities and other research institutions have identified this potential resulting from these characteristics, but also most business sectors are investigating the implications of this new technology (Zhao et al., 2016). Due to the enormous dynamics and the accompanying rapid development of the technology, research and economic interests are closely interdependent. While research institutions depend on the practical implementation of their research results, the business sector relies on the theoretical implications and the development of new fields of application.

However, there is still a significant disparity between the academic implications and the actual practical execution. Risius and Spohrer (2017) argue that research predominately focused on technological questions, rather than on application, value creation, and governance. Despite the great interest of the business sector to integrate blockchain technology into business processes, there is still a lack of answers to central questions regarding the meaningful and effective use of blockchain technology in practice. Consequently, the scarcity of adequate solutions concerning scalability, governance, security, and regulation-among other critical attributes-impedes the diffusion and adoption of the blockchain technology in field applications. This circumstance certainly has a significant influence on the reluctance of the German economy to adopt the blockchain technology. While the majority of German companies are aware of the potential benefits of crypto assets, up until now no financial resources have been made available for its implementation (PwC, 2018).

Irrespective of the rapid development at the technological level, a major challenge for the research community will be to also examine in more detail the application-related implications such as scalability, governance, integration with IoT, regulation, and security. This requires research institutions and industry to work closely together to address the following challenges collectively.

\section{GOVERNANCE}

Received: 26 October 2018 Accepted: 20 February 2019 Published: 15 March 2019

A particular challenge for architects designing blockchain applications is the availability of a wide range of configuration options and variants. The uncertainty about the configuration possibilities of blockchains is a consequence of the insufficient research and technological evaluation of the various possibilities (Xu et al., 2017). When configuring blockchains, there are different degrees of accessibility, authorization, and visibility that determine the private or public properties of the architecture. Due to the wide range of combinations, the research community will have to find 
appropriate solutions in this field to improve the classification of properties in order to facilitate the comparability between different blockchains.

\section{TECHNOLOGY}

Despite increasing research on blockchain-relevant topics over the last years, scalability challenges such as throughput and latency were largely omitted from the research work (Yli-Huumo et al., 2016). However, scalability is a central facilitator for the comprehensive adaptation of blockchain technology in the practical context, given that scalability of a system is decisive for the potential wide-spread diffusion of an application. Many scientific approaches already exist to solve the scaling problem (Zheng et al., 2017), but so far no solution has been found to be practicable for large-scale applications. Particularly on this subject, science must expand its efforts to enhance the scalability of blockchains to represent credible opposition to central systems.

Also, the advent of quantum computing poses a significant risk to the security of today's cryptographic infrastructure as it could break the security measures of digital signatures (Reyna et al., 2018) and thereby the foundation of DLT. Although quantum computing does not yet have a high technological readiness level, active research is being conducted in this area (Rodenburg and Pappas, 2017). To ensure the security of distributed ecosystems in the future, the research community will have to conduct research on security measures for an approaching post quantum era (Sababa, 2018).

\section{INTEGRATION WITH OFF-CHAIN-WORLD: IOT AND IDENTITY MANAGEMENT}

Though the characteristics of a blockchain make an IoT driven economy seem viable, the implementation still raises a number of outstanding challenges which have yet to be studied by the scientific community. Integration so far seems impractical because most blockchains are computationally intensive, involve high bandwidth overheads and delays (Dorri et al., 2017). The overarching challenges encompass difficulties in scalability, security, anonymity, data privacy, the further development of smart contracts and legal issues (Reyna et al., 2018). To solve these challenges, science will need to intensify research efforts on the overall application side.

Regarding the integration of sensors and machinery, scientific research on systems security has yet to be completed. A major challenge of integrating IoT with Blockchain is the reliability of the data collected by sensors and machinery. Blockchain can ensure that the data on the chain is immutable and that changes to a record within the network can be detected. However, if data is included that is already corrupt before it is added to the blockchain, the data remains corrupt (Reyna et al., 2018). To successfully integrate Blockchain with IoT, it will be essential to identify corrupted or false data prior to admission to the blockchain.

Sensors, for example, are the technical part of the off-chain world. But there is also identity management: human beings could store their identities on blockchain systems; be it with or without passports. Even more so, companies could be identified by putting their legal representatives or their hierarchical structures on a blockchain system. This way, identities could be re-used-instead of having to register case-by-case.

\section{REGULATION AND LAW}

Due to unresolved regulatory issues partly due to a neglect of science on this topic, a mass adoption of blockchain technology seems not practicable so far. Cermeño (2016) describes the present regulatory landscape as both immature and complex. Up to now, organizations lack the regulatory certainty necessary to implement blockchain applications with acceptable business risk. Thereby, the approval of governments and central banks is indispensable in this respect making blockchain technology fully adoptable. Science has to support the regulators to legally classify the blockchain technology in order to make the legal implications and amendments of the existing law feasible.

In the area of DLT and blockchain, a large amount of research has been made so far. However, it is questionable whether the thematic balance of the studies carried out is appropriate in order to advance the development of DLT and blockchain in a meaningful and sustainable way (Yli-Huumo et al., 2016). In the future, it will be important for the scientific community to find a healthy balance between theoretical basic and applicationspecific research. This will be the prerequisite for implementing theoretical research into practical applications. A second prerequisite for overcoming the aforementioned challenges is close cooperation between related areas of technology. In association with blockchain technology, especially the Industry 4.0 and the Internet of Things (IoT) have to be called into consideration. Each of these technology approaches focuses on transparency and decentralization, while at the same time facing a lack of scalability, interoperability, and data reliability. As digitalization progresses, these technologies will not only coexist but will increasingly converge. By strengthening and pooling collaboration to respond to each of these common central challenges, a basis will be provided for the exchange of theoretical basic and application-specific research that will substantially accelerate the progress and commercialization of the technologies.

\section{AUTHOR CONTRIBUTIONS}

PSa and PSc contributed conception and design of the study. PSa wrote the first draft of the manuscript. PSc wrote sections of the manuscript. All authors contributed to manuscript revision, read and approved the submitted version. 


\section{REFERENCES}

Cermeño, J. S. (2016). "Blockchain in financial services: regulatory landscape and future challenges for its commercial application," in BBVA Research Paper 16/20. (Economic Research Department).

Dorri, A., Kanhere, S. S., and Jurdak, R. (2017). "Towards an optimized blockchain for IoT," in Proceedings of the Second International Conference on Internet-ofThings Design and Implementation (New York, NY: ACM), 173-178.

PwC (2018). Blockchain in Financial Services-Mehr als nur ein Hype? Available online at: https://www.pwc.de/de/finanzdienstleistungen/blockchain-infinancial-services-2018.pdf

Reyna, A., Martín, C., Chen, J., Soler, E., and Díaz, M. (2018). On blockchain and its integration with IoT. Challenges and opportunities. Future Generat. Comput. Syst. 88, 173-190. doi: 10.1016/J.FUTURE.2018.05.046

Risius, M., and Spohrer, K. (2017). A Blockchain Research Framework. Bus. Inform. Syst. Eng. 59, 385-409. doi: 10.1007/s12599-017-0506-0

Rodenburg, B., and Pappas, S. P. (2017). Blockchain and Quantum Computing [Internet]. Princeton, NJ: Mitre. Available online at: https://www.mitre. org/sites/default/files/publications/17-4039-blockchain-and-quantumcomputing.pdf (Cited October 20, 2018).

Sababa, H. (2018). Cryptography in the Post-Quantum Era: The Evolution of Cryptography and Quantum Mechanics and Their Intersection in the Post-Quantum Era. University of Nicosia. Retrieved from: https:// www.researchgate.net/publication/322662995_Cryptography_in_the_PostQuantum_Era_The_evolution_of_cryptography_and_quantum_mechanics_ and_their_intersection_in_the_Post-Quantum_Era
Xu, X., Weber, I., Staples, M., Zhu, L., Bosch, J., Bass, L., et al. Rimba, P. (2017). A Taxonomy of Blockchain-Based Systems for Architecture Design. Available online at: http://design.inf.usi.ch/sites/default/files/biblio/icsa2017-blockchain.pdf

Yli-Huumo, J., Ko, D., Choi, S., Park, S., and Smolander, K. (2016). Where is current research on blockchain technology?-A systematic review. PLOS ONE 11:e0163477. doi: 10.1371/journal.pone.0 163477

Zhao, J. L., Fan, S., and Yan, J. (2016). Overview of business innovations and research opportunities in blockchain and introduction to the special issue. Finan. Innov. 2:28. doi: 10.1186/s40854-0160049-2

Zheng, Z., Xie, S., Dai, H.-N., Chen, X., and Wang, H. (2017). Blockchain Challenges and Opportunities: A Survey. Available online at: https://www. henrylab.net/wp-content/uploads/2017/10/blockchain.pdf

Conflict of Interest Statement: The authors declare that the research was conducted in the absence of any commercial or financial relationships that could be construed as a potential conflict of interest.

Copyright (C) 2019 Sandner and Schulden. This is an open-access article distributed under the terms of the Creative Commons Attribution License (CC BY). The use, distribution or reproduction in other forums is permitted, provided the original author(s) and the copyright owner(s) are credited and that the original publication in this journal is cited, in accordance with accepted academic practice. No use, distribution or reproduction is permitted which does not comply with these terms. 